\title{
LIMITS OF FUZZY RETRACTIONS OF FUZZY HYPERSPHERES AND THEIR FOLDINGS
}

\author{
A. E. EL-AHMADY
}

\begin{abstract}
This article introduces the types of fuzzy retractions of fuzzy hyperspheres $\tilde{S}^{n} \subset$ $R^{n+1}$ and the corresponding induced fuzzy retractions of the two fuzzy systems $\bigcup \overline{\tilde{S}}^{n}, \bigcup \underline{\tilde{S}}^{n}$ when the fuzzification is regular or irregular. Also, the fuzzy folding of fuzzy hyperspheres $\tilde{S}^{n}$ into itself and the induces two chains of fuzzy folding of $\bigcup \tilde{S}^{n}, \bigcup \underline{\tilde{S}}^{n}$. The relations between the fuzzy folding and fuzzy retraction are obtained. The limits of fuzzy retractions and fuzzy folding are achieved.
\end{abstract}

\section{Introduction}

To obtain the main results we will introduce the following definitions.

1. Fuzzy sphere $[2,5]$. If we have a fuzzy graph $\tilde{G}$ which is homeomorphic to a sphere $\tilde{S}$, this sphere is called a fuzzy sphere and the degree of fuzziness under this homeomorphism is constant.

2. A map $f: \tilde{S} \rightarrow \tilde{S}$, where $\tilde{S}$ is a fuzzy sphere, is said to be an isometric folding of $\tilde{S}$ into itself, iff for any piecewise fuzzy geodesic path $\gamma: J \rightarrow \tilde{S}$ the induced path $f \circ \gamma: J \rightarrow \tilde{S}$ is a piecewise fuzzy geodesic and of the same length as $\gamma$, where $J=[0,1]$. If $f$ does not preserve lengths, then $f$ is a topological folding of fuzzy sphere $[2,3,6]$.

3. A fuzzy subset $(\underset{\sim}{A}, \mu)$ of a fuzzy manifold $(\underset{\sim}{M}, \mu)$ is called a fuzzy retraction of $(M, \mu)$ if there exist a continuous map [2, 3, 8]. $\tilde{r}:(M, \mu) \rightarrow(\underset{\sim}{A}, \mu)$. Such that

$$
\tilde{r}(a, \mu(a))=(a, \mu(a)), \quad \forall a \in A, \mu \in[0,1] .
$$

4. The limit of the folding of a fuzzy manifold $\tilde{M}^{n}$ of dimension $n$ into itself is either a fuzzy manifold $\tilde{M}^{n-1}$ of dimension $n-1$, if $\forall a_{1}, a_{2} \in \tilde{M}^{n}, \mu\left(a_{1}\right)=\mu\left(a_{2}\right)$, or a fuzzy manifold of the same dimension $n$, if $\forall a_{1}, a_{2} \in \tilde{M}^{n}, \mu\left(a_{1}\right) \neq \mu(a 2), \mu \in[0,1][7]$.

Theorem 1. The fuzzy retractions of the fuzzy hypersphere $\tilde{S}^{n} \subset R^{n+1}$ induces two chains of fuzzy retractions of the two fuzzy systems of fuzzy hyperspheres $\bigcup \overline{\tilde{S}}^{n}$ and $\bigcup \underline{\tilde{S}}^{n}$.

Received and revised June 29, 2004.

2000 Mathematics Subject Classification. 51H10, 57N20.

Key words and phrases. Fuzzy hypersphere, fuzzy retraction, folding. 
Proof. Let $\tilde{S}^{n} \subset R^{n+1}$ be a fuzzy hyperspheres and $P_{i} \in \tilde{S}^{n}$, then there are induced nested 2-chains of $n$-pure fuzzy spheres $\bigcup \overline{\tilde{S}}_{i}^{n}$ and $\bigcup \underline{\tilde{S}}_{i}^{n}$. The fuzzy parametric equation of the fuzzy hypersphere $\tilde{S}^{n}$ is given by $[1,4]$.

$$
\begin{gathered}
\tilde{R}=\left(\left(r \cos \psi_{1} \prod_{K=1}^{n-1} \sin \psi_{K+1}, r \prod_{K=1}^{n} \sin \psi_{K}, \ldots, r \cos \psi_{i-1} \prod_{K=i}^{n} \sin \psi_{K}, \ldots, r \cos \psi_{n}\right), \mu\right), \\
i=3,4,5, \ldots, n, \quad \mu \in[0,1] .
\end{gathered}
$$

(a) Now, if $r_{i}<r, i=1,2,3, \ldots, \infty$. On $\bigcup \overline{\tilde{S}}_{i}^{n}$ the fuzzy retraction $\tilde{R}_{1}, \tilde{R}_{1}:\left(\tilde{S}^{n}-\right.$ $\left.\left\{\tilde{P}_{i 1}\right\}\right) \rightarrow \tilde{S}^{n-1}$, this retraction induces two fuzzy retractions $\overline{\tilde{R}}_{1}:\left(\overline{\tilde{S}}_{i}^{n}-\left\{\overline{\tilde{P}}_{i 1}\right\}\right) \rightarrow \overline{\tilde{S}}^{n-1}$, $\frac{\tilde{R}_{1}}{\tilde{S}^{n-1}}\left(\underline{\tilde{S}}_{i}^{n}-\left\{\underline{\tilde{P}}_{i 1}\right\}\right) \rightarrow \underline{\tilde{S}}_{i}^{n-1}$, where the fuzzy parametric equation of the fuzzy hyperspheres

$$
\tilde{R}_{1}=\left(\left(r_{1} \prod_{K=1}^{n-1} \sin \psi_{K+1}, 0, \ldots, r_{1} \cos \psi_{i-1} \prod_{K=i}^{n} \sin \psi_{K}, \ldots, r_{1} \cos \psi_{n}\right), \mu\right), r_{1}<r .
$$

Also, the fuzzy retraction $\tilde{R}_{2}:\left\{\tilde{S}^{n-1}-\left\{\tilde{P}_{i 2}\right\}\right\} \rightarrow \tilde{S}^{n-2}$,

$$
\begin{aligned}
& \tilde{R}_{2}=\left(\left(r_{2} \prod_{K=1}^{n-1} \sin \psi_{K+1}, 0, \ldots, r_{2} \cos \psi_{i-1} \prod_{K=i}^{n} \sin \psi_{K}, \ldots, r_{2} \cos \psi_{n}\right), \mu\right) \\
& r_{2}<r_{1}<r, \quad i=4,5,6, \ldots, n .
\end{aligned}
$$

This retraction induces two fuzzy retractions $\overline{\tilde{R}}_{2}:\left\{\overline{\widetilde{S}}_{i}^{n-1}-\left\{\overline{\tilde{P}}_{i 2}\right\}\right\} \rightarrow \overline{\tilde{S}}_{i}^{n-2}, \underline{\tilde{R}}_{2}:\left\{\underline{\tilde{S}}_{i}^{n-1}-\right.$ $\left.\left\{\underline{\tilde{P}}_{i 2}\right\}\right\} \rightarrow \underline{\tilde{S}}_{i}^{n-2}$. Also, the fuzzy retraction $\tilde{R}_{3}$, where $\tilde{R}_{3}:\left\{\overline{\tilde{S}}^{n-2}-\left\{\overline{\tilde{P}}_{i 3}\right\}\right\} \rightarrow \underline{\tilde{S}}^{n-3}$, This retraction induces two fuzzy retractions $\overline{\tilde{R}}_{3}:\left\{\overline{\tilde{S}}_{i}^{n-2}-\left\{\overline{\tilde{P}}_{i 3}\right\}\right\} \rightarrow \overline{\tilde{S}}_{i}^{n-3}, \underline{\tilde{R}}_{3}:\left\{\underline{\tilde{S}}_{i}^{n-2}-\right.$ $\left.\left\{\underline{\tilde{P}}_{i 3}\right\}\right\} \rightarrow \underline{\tilde{S}}_{i}^{n-3}$, where the fuzzy parametric equation of the fuzzy retraction of fuzzy hypersphere $\tilde{S}^{n-3}$ is

$$
\begin{array}{r}
\tilde{R}_{3}=\left(\left(r_{3} \prod_{K=1}^{n-1} \sin \psi_{K+1}, 0, \ldots, r_{3} \cos \psi_{i-1} \prod_{K=i}^{n} \sin \psi_{K}, \ldots, r_{3} \cos \psi_{n}\right), \mu\right) \\
r_{3}<r_{2}<r_{1}<r, \quad i=5,6, \ldots, n .
\end{array}
$$

Hence, the fuzzy retraction $\tilde{R}_{n-1}, \tilde{R}_{n-1}:\left(\tilde{S}^{2}-\left\{\tilde{P}_{i(n-1)}\right\}\right) \rightarrow \tilde{S}^{1}$, induces two fuzzy retractions

$$
\overline{\tilde{R}}_{n-1}:\left(\overline{\tilde{S}}_{i}^{2}-\left\{\overline{\tilde{P}}_{i(n-1)}\right\}\right) \rightarrow \overline{\tilde{S}}_{i}^{1}, \quad \underline{\tilde{R}}_{n-1}:\left(\underline{\tilde{S}}_{i}^{2}-\left\{\underline{\tilde{P}}_{i(n-1)}\right\}\right) \rightarrow \underline{\tilde{S}}_{i}^{1},
$$

where the fuzzy parametric equation of the fuzzy spheres $\tilde{S}^{2}$ is given by

$$
\begin{aligned}
\tilde{R}_{n-1}=\left(\left(r_{n-1} \cos \psi_{1} \sin \psi_{2}, r_{n-1} \sin \psi_{1} \sin \psi_{2}, r_{n-1} \cos \psi_{2}\right), \mu\right), \\
r_{n-1}<r_{n-2}<r_{n-3}<\cdots<r_{3}<r_{2}<r_{1}<r .
\end{aligned}
$$


The fuzzy retraction $\tilde{R}_{n}$, where $\tilde{R}_{n}:\left\{\tilde{S}^{1}-\left\{\tilde{P}_{i n}\right\}\right\} \rightarrow \tilde{S}^{0}$, induces two fuzzy retractions

$$
\overline{\tilde{R}}_{n}:\left\{\overline{\tilde{S}}_{i}^{1}-\left\{\overline{\tilde{P}}_{i n}\right\}\right\} \rightarrow \overline{\tilde{S}}_{i}^{0}, \quad \underline{\tilde{R}}_{n}:\left\{\underline{\tilde{S}}_{i}^{1}-\left\{\underline{\tilde{P}}_{i n}\right\}\right\} \rightarrow \underline{\tilde{S}}_{i}^{0},
$$

where the fuzzy parametric equation of the fuzzy circle is given by:

$$
\tilde{R}_{n}=\left(\left(r_{n} \sin \psi_{2}, 0, r_{n} \cos \psi_{2}\right), \mu\right), \quad r_{n}<r_{n-1}<r_{n-2}<\cdots<r_{3}<r_{2}<r_{1}<r .
$$

(a) If $r_{i}>r, i=1,2,3, \ldots, \infty$ on $\bigcup \underline{\tilde{S}}_{i}^{n}$. We have the same results in (a), see Figure 1

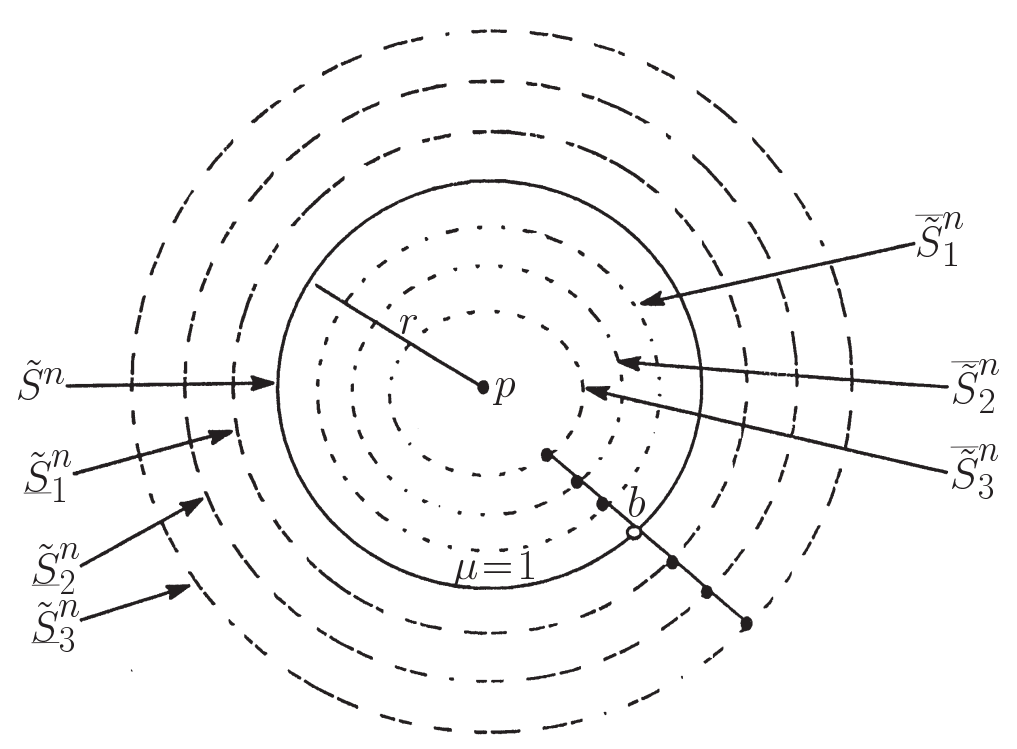

Figure 1.

Theorem 2. The folding of the fuzzy hypersphere $\tilde{S}^{n} \subset R^{n+1}$ into itself induces two chains of foldings $\bigcup \overline{\tilde{S}}^{n}$ and $\bigcup \underline{\tilde{S}}^{n}$ which is a type of fuzzy retractions.

Proof. Assume $\tilde{f}_{1}: \tilde{S}^{n} \rightarrow \tilde{S}^{n}$ be a fuzzy folding from $\tilde{S}^{n}$ into $\tilde{S}^{n}$ such that $\tilde{f}_{1}\left(\tilde{S}^{n}\right) \neq$ $\tilde{S}^{n}$. This folding induces 2-chains of fuzzy foldings $\overline{\tilde{f}}_{1}: \overline{\tilde{S}}^{n} \rightarrow \overline{\tilde{S}}^{n}$ such that $\overline{\tilde{f}}_{1}:\left(\overline{\tilde{S}}^{n}\right) \neq \overline{\tilde{S}}^{n}$ and $\underline{\tilde{f}}_{1}: \underline{\tilde{S}}^{n} \rightarrow \underline{\tilde{S}}^{n}$ such that $\underline{\tilde{f}}_{1}:\left(\underline{\tilde{S}}^{n}\right) \neq \underline{\tilde{S}}^{n}$, where $\operatorname{dim} \tilde{f}_{1}=\operatorname{dim} \overline{\tilde{f}}_{1}=\operatorname{dim} \underline{\tilde{f}}_{1} \neq \operatorname{dim} \tilde{R}_{1}$. Then the fuzzy folding not conside with the fuzzy retraction. Also, let

$\tilde{f}_{2}: \tilde{f}_{1}\left(\tilde{S}^{n}\right) \rightarrow \tilde{f}_{1}\left(\tilde{S}^{n}\right), \quad$ this folding induces $\tilde{\tilde{f}}_{2}$ and $\underline{\tilde{f}}_{2}$ where

$\overline{\tilde{f}}_{2}: \overline{\tilde{f}}_{1}\left(\overline{\tilde{S}}^{n}\right) \rightarrow \overline{\tilde{f}}_{1}\left(\overline{\tilde{S}}^{n}\right), \quad \underline{\tilde{f}}_{2}: \underline{\tilde{f}}_{1}\left(\underline{\tilde{S}}^{n}\right) \rightarrow \underline{\tilde{f}}_{1}\left(\underline{\tilde{S}}^{n}\right)$,

$\tilde{f}_{3}: \tilde{f}_{2}\left(\tilde{f}_{1}\left(\tilde{S}^{n}\right)\right) \rightarrow \tilde{f}_{2}\left(\tilde{f}_{1}\left(\tilde{S}^{n}\right)\right)$, where

$\overline{\tilde{f}}_{3}: \overline{\tilde{f}}_{2}\left(\overline{\tilde{f}}_{1}\left(\overline{\tilde{S}}^{n}\right)\right) \rightarrow \overline{\tilde{f}}_{2}\left(\overline{\tilde{f}}_{1}\left(\overline{\tilde{S}}^{n}\right)\right)$ 


$$
\begin{aligned}
& \tilde{\tilde{f}}_{3}: \tilde{\tilde{f}}_{2}\left(\tilde{\tilde{f}}_{1}\left(\tilde{\tilde{S}}^{n}\right)\right) \rightarrow \underline{\tilde{f}}_{2}\left(\tilde{\tilde{f}}_{1}\left(\underline{\tilde{S}}^{n}\right)\right), \\
& \tilde{f}_{4}: \tilde{f}_{3}\left(\tilde{f}_{2}\left(\tilde{f}_{1}\left(\tilde{S}^{n}\right)\right)\right) \rightarrow \tilde{f}_{3}\left(\tilde{f}_{2}\left(\tilde{f}_{1}\left(\tilde{S}^{n}\right)\right)\right), \quad \text { where } \\
& \tilde{\tilde{f}}_{4}: \overline{\tilde{f}}_{3}\left(\overline{\tilde{f}}_{2}\left(\overline{\tilde{f}}_{1}\left(\overline{\tilde{S}}^{n}\right)\right)\right) \rightarrow \overline{\tilde{f}}_{3}\left(\overline{\tilde{f}}_{2}\left(\overline{\tilde{f}}_{1}\left(\overline{\tilde{S}}^{n}\right)\right)\right), \\
& \tilde{\tilde{f}}_{4}: \tilde{\tilde{f}}_{3}\left(\tilde{f}_{2}\left(\tilde{\tilde{f}}_{1}\left(\underline{\tilde{S}}^{n}\right)\right)\right) \rightarrow \tilde{\tilde{f}}_{3}\left(\tilde{\tilde{f}}_{2}\left(\tilde{\tilde{f}}_{1}\left(\underline{\tilde{S}}^{n}\right)\right)\right), \ldots, \\
& \tilde{\tilde{f}}_{n}: \tilde{f}_{n-1}\left(\tilde{f}_{n-2}\left(\tilde{f}_{n-3} \cdots \tilde{f}_{2}\left(\tilde{f}_{1}\left(\tilde{S}^{n}\right)\right) \cdots\right)\right) \rightarrow \tilde{f}_{n-1}\left(\tilde{f}_{n-2}\left(\tilde{f}_{n-3} \cdots \tilde{f}_{2}\left(\tilde{f}_{1}\left(\tilde{S}^{n}\right)\right) \cdots\right)\right) .
\end{aligned}
$$

Then the fuzzy folding induces two chains of fuzzy foldings

$$
\begin{aligned}
& \tilde{\tilde{f}}_{n}: \overline{\tilde{f}}_{n-1}\left(\overline{\tilde{f}}_{n-2}\left(\overline{\tilde{f}}_{n-3} \cdots \overline{\tilde{f}}_{2}\left(\overline{\tilde{f}}_{1}\left(\overline{\tilde{S}}^{n}\right)\right) \cdots\right)\right) \rightarrow \overline{\tilde{f}}_{n-1}\left(\overline{\tilde{f}}_{n-2}\left(\overline{\tilde{f}}_{n-3} \cdots \overline{\tilde{f}}_{2}\left(\overline{\tilde{f}}_{1}\left(\overline{\tilde{S}}^{n}\right)\right) \cdots\right)\right), \\
& \underline{\tilde{f}}_{n}: \underline{\tilde{f}}_{n-1}\left(\underline{\tilde{f}}_{n-2}\left(\tilde{\tilde{f}}_{n-3} \cdots \underline{\tilde{f}}_{2}\left(\underline{\tilde{f}}_{1}\left(\underline{\tilde{S}}^{n}\right)\right) \cdots\right)\right) \rightarrow \underline{\tilde{f}}_{n-1}\left(\underline{\tilde{f}}_{n-2}\left(\underline{\tilde{f}}_{n-3} \cdots \underline{\tilde{f}}_{2}\left(\tilde{f}_{1}\left(\underline{\tilde{S}}^{n}\right)\right) \cdots\right)\right) .
\end{aligned}
$$

Lemma 1. The limit of the conditional fuzzy folding of fuzzy hypersphere $\tilde{S}^{n}$ induces 2-chains of limits of fuzzy foldings which is equivalent to the 2-chains of fuzzy retractions in Theorem (1).

Proof. From Theorem (2) if $\tilde{f}_{n}: \tilde{S}^{n} \rightarrow \tilde{S}^{n}$ be a fuzzy folding from $\tilde{S}^{n}$ into $\tilde{S}^{n}$ such that $\tilde{f}_{n} / \tilde{S}^{n-1}=\tilde{S}^{n-1}$, then $\lim _{n \rightarrow \infty}\left(\tilde{f}_{n} / \tilde{S}^{n-1}\right)\left(\tilde{S}^{n}\right)=\tilde{S}^{n-1}$.

This folding induces 2-chains of fuzzy folding $\overline{\tilde{f}}_{n}: \overline{\tilde{S}}^{n} \rightarrow \overline{\widetilde{S}}^{n}$ such that $\overline{\tilde{f}}_{n} / \overline{\tilde{S}}^{n-1}=\overline{\tilde{S}}^{n-1}$, $\underline{\tilde{f}}_{n}: \underline{\tilde{S}}^{n} \rightarrow \underline{\tilde{S}}^{n}$ such that $\underline{\tilde{f}}_{n} / \underline{\tilde{S}}^{n-1}=\underline{\tilde{S}}^{n-1}$,

$$
\lim _{n \rightarrow \infty}\left(\overline{\tilde{f}}_{n} / \overline{\tilde{S}}^{n-1}\right)\left(\overline{\tilde{S}}^{n}\right)=\overline{\tilde{S}}^{n-1} \text { and } \quad \lim _{n \rightarrow \infty} \underline{\tilde{f}}_{n} / \underline{\tilde{S}}^{n-1}\left(\underline{\tilde{S}}^{n}\right)=\underline{\tilde{S}}^{n-1} \text {. }
$$

Then the limit of this type of conditonal fuzzy folding conside with the fuzzy retractions of Theorem (1).

If the fuzzification is not distributed regular on the fuzzy hypersphere $\tilde{S}^{n}$, then we have the Figure 2.

The interior are homeomorphic to fuzzy hypersphere $\overline{\tilde{S}}^{n}$ and $\underline{\tilde{S}}^{n}$. Consequently we will have to the following theorem.

Theorem 3. The fuzzy retraction of fuzzy hyperspheres $\tilde{S}^{n} \subset R^{n+1}$ induces two chains of fuzzy retractions of the two fuzzy systems of fuzzy manifolds homeomorphic to fuzzy spheres $\bigcup \overline{\tilde{S}}^{n}$ and $\bigcup \underline{\tilde{S}}^{n}$.

Proof. There are two types of fuzzy retractions

(i) The fuzzy retraction with fixed point $\tilde{P}$ we will arrive to two systems of fuzzy retractions which are immersed as in Theorem (1).

(ii) The fuzzy retraction with removing the fixed point $\tilde{p}$ and its antipodal point $\tilde{p}$, we will have two systems of fuzzy hypermanifolds homeomorphic to types of fuzzy spheres $\overline{\tilde{S}}^{n-1}$ and $\underline{\tilde{S}}^{n-1}$ which are not immersed and consequently we obtain the two chains like Theorem (1). 


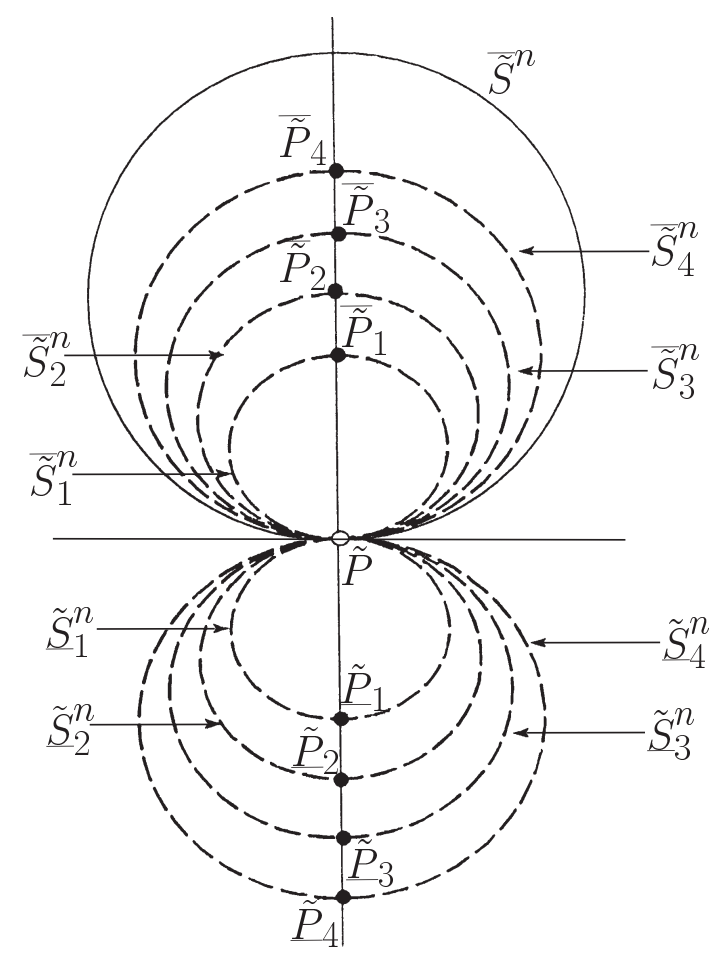

Figure 2 .

Theorem 4. The folding of the fuzzy hyperspheres $\tilde{S}^{n}$ of the second type into itself induces two chains of fuzzy folding of two systems of fuzzy manifolds homeomorphic to $\bigcup \overline{\tilde{S}}^{n}$ and $\bigcup \underline{\tilde{S}}^{n}$.

Proof. From Theorem (2) the proof is clear.

Lemma 2. The limit of conditional fuzzy folding of fuzzy hyperspheres $\tilde{S}^{n}$ induces 2-chains of limits of fuzzy folding of fuzzy hypermanifolds homeomorphic to $\bigcup \overline{\tilde{S}}^{n}$ and U $\underline{\tilde{S}}^{n}$.

The system of nested $n$-inside spheres can be deformed by any outer effect on the physical character to be given in Figure 3.

Lemma 3. The fuzzy retraction of any fuzzy deformed system in theorems (1) and (2) can not be discussed analytically but their fuzzy retraction are homeomorphic to $\bigcup \overline{\tilde{S}}_{j}^{n}$ and $\bigcup \underline{\tilde{S}}_{j}^{n}$. See Figure 3 . 

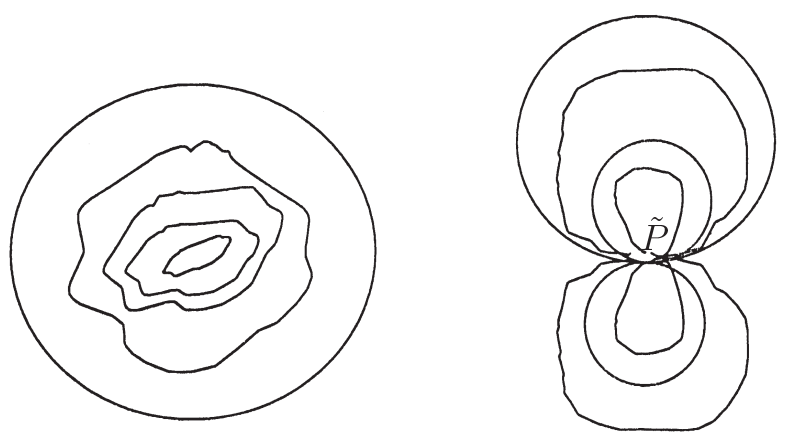

Figure 3.

Theorem 5. If the fuzzy retraction of the fuzzy hypersphere $\tilde{S}^{n} \subset R^{n+1}$ is $\tilde{r}: \tilde{S}^{n}-$ $\left(\tilde{P}_{n}\right) \rightarrow \tilde{S}^{n-1}$ and the fuzzy folding of $\tilde{S}^{n}-\left(\tilde{P}_{n}\right)$ into itself is $\tilde{f}: \tilde{S}^{n}-\left(\tilde{P}^{n}\right) \rightarrow \tilde{S}^{n}-\left(\tilde{P}^{n}\right)$, then there are induces 2 -chains of fuzzy retractions and foldings such that the following diagrams are commutatives.

Proof. Let the fuzzy retraction of $\tilde{S}^{n}$ is defined by $\tilde{r}_{1}: \tilde{S}^{n}-\left(\tilde{P}_{n}\right) \rightarrow \tilde{S}^{n-1}$ and the fuzzy folding of $\tilde{S}^{n}, \tilde{S}^{n-1}$ are given by $\tilde{f}_{1}: \tilde{S}^{n}-\left(\tilde{P}_{n}\right) \rightarrow \tilde{S}^{n}-\left(\tilde{P}^{n}\right), \tilde{f}_{2}: \tilde{S}^{n-1} \rightarrow \tilde{S}^{n-1}$. Also, $\tilde{r}_{2}: \tilde{f}_{1}\left(\tilde{S}^{n}-\left(\tilde{P}_{n}\right)\right) \rightarrow \tilde{S}^{n-1}$. Then, there are induced 2-chains of fuzzy retractions and foldings are given by

$$
\begin{array}{ll}
\overline{\tilde{r}}_{1}: \overline{\tilde{S}}^{n}-\left(\overline{\tilde{P}}_{n}\right) \rightarrow \overline{\tilde{S}}^{n-1}, & \underline{\tilde{r}}_{1}: \underline{\tilde{S}}^{n}-\left(\underline{\tilde{P}}_{n}\right) \rightarrow \underline{\tilde{S}}^{n-1}, \\
\tilde{\tilde{f}}_{1}: \overline{\tilde{S}}^{n}-\left(\overline{\tilde{P}}_{n}\right) \rightarrow \overline{\tilde{S}}^{n}-\left(\overline{\tilde{P}}_{n}\right), & \tilde{\tilde{f}}_{2}: \overline{\tilde{S}}^{n-1} \rightarrow \overline{\tilde{S}}^{n-1}, \\
\tilde{f}_{1}: \underline{\tilde{S}}^{n}-\left(\underline{\tilde{P}}_{n}\right) \rightarrow \underline{\tilde{S}}^{n}-\left(\underline{\tilde{P}}_{n}\right), & \underline{\tilde{f}}_{2}: \underline{\tilde{S}}^{n-1} \rightarrow \underline{\tilde{S}}^{n-1} .
\end{array}
$$

Hence, the following diagrams are commutatives

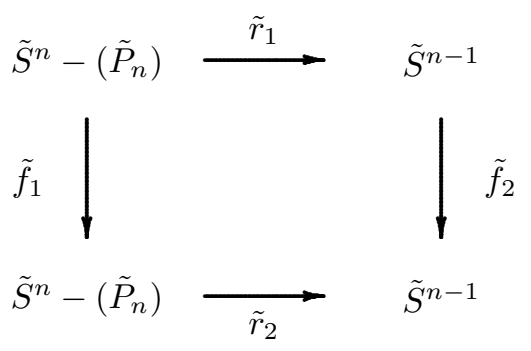




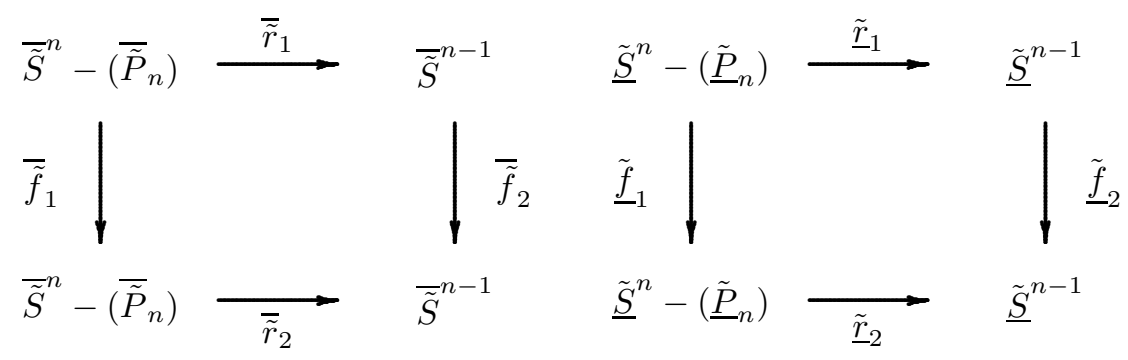

Lemma 4. The generalization of Theorem (5) represented by the following chains $\tilde{r}_{i+1} \circ \tilde{f}_{i}=\tilde{f}_{i+1} \circ \tilde{r}_{i}, \overline{\tilde{r}}_{i+1} \circ \overline{\tilde{f}}_{i}=\overline{\tilde{f}}_{i+1} \circ \overline{\tilde{r}}_{i}$, and $\underline{\tilde{r}}_{i+1} \circ \underline{\tilde{f}}_{i}=\underline{\tilde{f}}_{i+1} \circ \underline{\tilde{r}}_{i}, i=1,2,3, \ldots, n$.

Lemma 5. The relation between the fuzzy retraction and the limit of the fuzzy foldings discussed from the following commutative diagram

$$
\begin{gathered}
\tilde{S}^{n}-\left(\tilde{P}_{n}\right) \stackrel{\tilde{r}_{1}}{\longrightarrow} \tilde{S}^{n-1}-\left(\tilde{P}_{n-1}\right) \\
\left.\lim _{m \rightarrow \infty} \tilde{f}_{m}\right|_{m \rightarrow \infty} \lim _{m+1} \tilde{f}_{m+\infty} \tilde{r}_{j} \tilde{S}^{n-2}-\left(\tilde{P}_{n-2}\right)
\end{gathered}
$$

and the corresponding relation between the two chains of fuzzy retractions and the limit of fuzzy foldings are described from the two induces diagrams

$$
\begin{gathered}
\overline{\tilde{S}}^{n}-\left(\overline{\tilde{P}}_{n}\right) \stackrel{\overline{\tilde{r}}_{1}}{\longrightarrow} \overline{\tilde{S}}^{n-1}-\left(\overline{\tilde{P}}_{n-1}\right) \\
\lim _{m \rightarrow \infty} \overline{\tilde{f}}_{m} \mid \begin{array}{|}
\lim _{m \rightarrow \infty} \overline{\tilde{f}}_{m+1} \\
\overline{\tilde{S}}^{n-1}-\left(\overline{\tilde{P}}_{n-1}\right) \frac{\overline{\tilde{r}}_{j}}{\longrightarrow}-\overline{\tilde{S}}^{n-2}-\left(\overline{\tilde{P}}_{n-2}\right)
\end{array}
\end{gathered}
$$




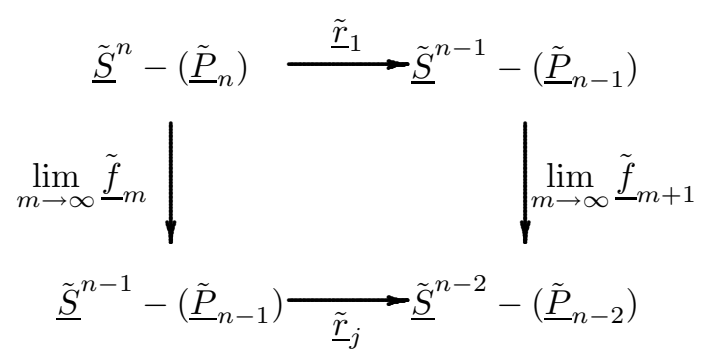

Lemma 6. The end of the limits of the fuzzy foldings and fuzzy retractions of the system will be

$$
\begin{array}{cc}
\lim _{m \rightarrow \infty} \tilde{S}_{m_{1}}-\left(\tilde{P}_{2}\right) \stackrel{\tilde{r}_{K}}{\longrightarrow} & \tilde{S}^{1}-\left(\tilde{P}_{1}\right) \\
\tilde{S}^{1}-\left(\tilde{P}_{1}\right) \stackrel{\lim _{m \rightarrow \infty}}{\frac{\tilde{r}_{m_{2}}}{\longrightarrow}} & 0
\end{array}
$$

Also, the induced relation between the two chains of the end of limits of fuzzy foldings and fuzzy retractions of the system are defined by

$$
\begin{aligned}
& \overline{\tilde{S}}^{2}-\left(\tilde{\tilde{P}}_{2}\right) \stackrel{\overline{\tilde{r}}_{K}}{\longrightarrow} \overline{\tilde{S}}^{1}-\left(\tilde{\tilde{P}}_{1}\right) \\
& \underline{\tilde{S}}^{2}-\left(\underline{\tilde{P}}_{2}\right) \stackrel{\underline{\tilde{r}}_{K}}{\longrightarrow} \underline{\tilde{S}}^{1}-\left(\underline{\tilde{P}}_{1}\right) \\
& \lim _{m \rightarrow \infty} \overline{\tilde{f}}_{m_{1}} \\
& \mid \lim _{m \rightarrow \infty} \overline{\tilde{f}}_{m_{2}} \\
& \lim _{m \rightarrow \infty} \tilde{\tilde{f}}_{m_{1}} \\
& \mid \lim _{m \rightarrow \infty} \tilde{f}_{m_{2}} \\
& \overline{\tilde{S}}^{1}-\left(\overline{\tilde{P}}_{1}\right) \underset{\overline{\tilde{r}}_{J}}{\longrightarrow} \\
& \underline{\tilde{S}}^{1}-\left(\underline{\tilde{P}}_{1}\right) \underset{\underline{\tilde{x}}_{J}}{\longrightarrow} 0
\end{aligned}
$$

\section{Applications}

1. Consider the flow of the fluid inside a tube, let the velocity of the fluid takes a maximum at $\mu=1$ in the mid of medium and the velocity round this line is symetric, at the edge of the tube the velocity of the fluid $=0=\mu$.

The decreasing or increasing of the flow of the fluid represented a type of retraction or a type of floding respectively.

2. Consider the ideal dose of radiation must be directed on the form of the fuzzy sphere 
$\tilde{S}^{n}$ and the dose on $\overline{\widetilde{S}}^{n}$ equal the dose on $\underline{\tilde{S}}^{n}$. The dose decreased if $\underline{r}_{i} \rightarrow 0$ or $r \rightarrow \infty$ the regular dose and irregular dose of radiation represented as $\mu$ regular on $\tilde{S}^{n}$ or irregular on $\tilde{S}^{n}$. Also, the decreasing in the two cases consider as the retraction discussed in this paper.

\section{References}

[1] A. E. El-Ahmady, The deformation retract and topological folding of Buchdahi space, Periodica Mathematica Hungarica 28(1994), 19-30.

[2] A. E. El-Ahmady, Fuzzy Lobachevskian space and its folding, The Journal of Fuzzy Mathematics 12 (2004), 255-260.

[3] A. E. El-Ahmady and H. M. Shamara, Fuzzy deformation retract of fuzzy horospheres, Indian J. Pure Appel. Math. 32(2001), 1501-1506, October.

[4] Afwat Abd El-Megid, M. EL-Ghoul and A. E. El-Ahmady, The deformation retract of hypersphere $S^{n}$ and its topological folding, Second Conference on Oper. Rec. and its Military Applications, BOR-10(1987), 856-862, Egypt.

[5] M. El-Ghoul, Fuzzy retraction and folding of fuzzy oriental compact manifolds, Fuzzy Sets and Systems 105(1999), 159-163, Germany.

[6] M. El-Ghoul and H. M. Shamara, Folding of some types of fuzzy manifolds and their retractions, fuzzy Sets and Systems 97(1998), 387-391, Germany.

[7] M. El-Ghoul and H. M. Shamara, The limit of the folding of some types of fuzzy manifolds, International Fuzzy Mathematics Institute, Los Angeles 7(1999), U.S.A.

[8] M. El-Ghoul, H. El-Zohny and S. Radwan, Deformation of some fuzzy manifolds and its folding, International Fuzzy Mathematics Institute, Los Angeles 9(2001), U.S.A.

Department of Mathematics, Faculty of Science, Tanta University, Tanta, Egypt. 